\title{
Effects of inhalation of low-dose nitrite or carbon monoxide on post-reperfusion mitochondrial function and tissue injury in hemorrhagic shock swine
}

Håkon Haugaa ${ }^{1,2,3^{*}}$, Hernando Gómez ${ }^{1,7}$, Donald R Maberry ${ }^{1}$, Andre Holder ${ }^{1}$, Olufunmilayo Ogundele ${ }^{1}$, Ana Maria B Quintero ${ }^{3}$, Daniel Escobar ${ }^{3}$, Tor Inge Tønnessen ${ }^{2,3}$, Hannah Airgood ${ }^{5}$, Cameron Dezfuliann, Elizabeth Kenny ${ }^{5}$, Sruti Shiva ${ }^{6}$, Brian Zuckerbraun ${ }^{4}$ and Michael R Pinsky ${ }^{1}$

\begin{abstract}
Introduction: Tissue reperfusion following hemorrhagic shock may paradoxically cause tissue injury and organ dysfunction by mitochondrial free radical expression. Both nitrite and carbon monoxide (CO) may protect from this reperfusion injury by limiting mitochondrial free radial production. We explored the effects of very small doses of inhaled nitrite and CO on tissue injury in a porcine model of hemorrhagic shock.

Methods: Twenty pigs (mean wt. $30.6 \mathrm{~kg}$, range 27.2 to $36.4 \mathrm{~kg}$ ) had microdialysis catheters inserted in muscle, peritoneum, and liver to measure lactate, pyruvate, glucose, glycerol, and nitrite. Nineteen of the pigs were bled at a rate of $20 \mathrm{ml} / \mathrm{min}$ to a mean arterial pressure of $30 \mathrm{mmHg}$ and kept between 30 and $40 \mathrm{mmHg}$ for 90 minutes and then resuscitated. One pig was instrumented but not bled (sham). Hemorrhaged animals were randomized to inhale nothing (control, $n=7$ ), 11 mg nitrite (nitrite, $n=7)$ or 250 ppm CO $(C O, n=5)$ over 30 minutes before fluid resuscitation. Mitochondrial respiratory control ratio was measured in muscle biopsies. Repeated measures from microdialysis catheters were analyzed in a random effects mixed model.

Results: Neither nitrite nor CO had any effects on the measured hemodynamic variables. Following inhalation of nitrite, plasma, but not tissue, nitrite increased. Following reperfusion, plasma nitrite only increased in the control and CO groups. Thereafter, nitrite decreased only in the nitrite group. Inhalation of nitrite was associated with decreases in blood lactate, whereas both nitrite and CO were associated with decreases in glycerol release into peritoneal fluid. Following resuscitation, the muscular mitochondrial respiratory control ratio was reduced in the control group but preserved in the nitrite and CO groups.
\end{abstract}

Conclusions: We conclude that small doses of nebulized sodium nitrite or inhaled CO may be associated with intestinal protection during resuscitation from severe hemorrhagic shock.

\footnotetext{
* Correspondence: hakon.haugaa@medisin.uio.no

'Department of Critical Care Medicine, Cardiopulmonary Research Laboratory,

University of Pittsburgh, 3501 Fifth Avenue, Pittsburgh, PA 15260, USA

2Department of Emergencies and Critical Care, Oslo University Hospital,

Sognsvannsveien 27 0424, Oslo, Norway

Full list of author information is available at the end of the article
} 


\section{Introduction}

The immediate restoration of blood pressure, cardiac output and oxygen-carrying capacity of patients in hemorrhagic shock is the fundamental goal of acute resuscitation. Adequate oxygen delivery to the tissues is dependent on the presence of an adequate perfusion pressure, and it is known that arterial pressure is strongly related to outcome. Patients experiencing blunt trauma who present with systolic hypotension have three times higher mortality than those who present with a normal blood pressure [1]. Furthermore, preemptive optimization of perfusion guided by oxygen delivery before surgery in high-risk patients results in a significant decrease in the rate of complications and length of stay, and once perfusion pressure is restored, optimization of cardiac output and oxygen-carrying capacity (oxygen delivery) appear beneficial [2-4]. However, reperfusion of ischemic tissue can induce reperfusion injury potentially leading to organ dysfunction and death [5].

The fundamental assumption in resuscitation physiology is that shock represents inadequate perfusion of the tissues to meet their metabolic demand and that rapid restoration of macrocirculatory perfusion pressure and blood flow will reverse this hypoperfusion, minimizing tissue injury and promoting recovery. However, it is unknown how resuscitation based on macrohemodynamic parameters impacts tissue wellness in the setting of hemorrhagic shock. Methods to quantify regional and local microcirculatory and oxygenation status have consistently shown a disconnection between macrohemodynamic parameters and tissues oxygenation and function. Mesquida et al. showed that tissue oxygen saturation measured in the thenar eminence by near-infrared spectroscopy had a weak correlation to mixed venous oxygen saturation $\left(\mathrm{SvO}_{2}\right)$ [6]. More importantly, Hernandez et al. recently showed that dobutamine-induced increases in macrocirculatory parameters did not translate into an improved microcirculatory flow or an improved organ system function in septic patients [7]. Thus, it is unclear if manipulating the macrocirculation will proportionally benefit the microcirculation after the development of tissue ischemia.

Nitric oxide (NO) is a gaseous, short half-life molecule typically administered as continuous inhalation to ventilated neonate and adult patients. The relatively expensive and cumbersome administration has restricted the utilization of NO to critical care patients. However, systemic NO effects can also be obtained by administration of the sodium nitrite $\left(\mathrm{NaNO}_{2}\right)$ [8]. Nitrite may be administered intravenously or nebulized as inhalation. In the body, it is reduced to NO by NO reductases such as xanthine oxidoreductase, aldehyde oxidase, deoxyhemoglobin, deoxymyoglobin, and cytochrome c oxidase $[8,9]$.
Mitochondrial free radical production and calcium poisoning play significant roles in reperfusion injury [5]. Although the primary function of mitochondria is to convert energy derived from nutrients into adenosine triphosphate (ATP), they also produce superoxide as a byproduct of electron transport and oxygen consumption. Reactive oxygen species (ROS) and reactive nitrogen species (RNS) can be generated by superoxide dismutation and reaction with $\mathrm{NO}$, respectively. In the setting of ischemia ROS/RNS production increases as electrons frequently leak from the injured electron transport change before reaching cytochrome c oxidase, potentially causing cell damage, inducing apoptosis, necrosis or both [10]. During ischemia, pharmacological preconditioning prior to resuscitation with nitrite or carbon monoxide $(\mathrm{CO})$ can prevent this 'uncontrolled' production of free radicals by partially blocking complex I [11-14] and complex III [15] of the electron transport chain. Potentially, these small molecules may protect the cell from reperfusion injury. Based on previous studies showing that beneficial effects of nitrite and $\mathrm{CO}$ may be obtained with very small doses [15-17], we therefore hypothesized that exogenous administration of low-dose $\mathrm{NaNO}_{2}$ or CO by inhalation before resuscitation would improve electron handling by the mitochondrial electron transport chain, and would decrease tissue injury as measured in the extracellular space through microdialysis catheters [18].

\section{Material and methods}

\section{Animal preparation and surgical procedure}

All experiments were performed in accordance with the United States National Institutes of Health guidelines under protocols approved by the Institutional Animal Care and Use Committee of the University of Pittsburgh (protocol No. 13061614). Twenty Yorkshire Durock pigs (average weight (wt.) of $30.6 \mathrm{~kg}$, range 27.2 to $36.4 \mathrm{~kg}$ ) were acclimatized in the animal facility for at least seven days prior to study. The swine were fasted overnight but with free access to water prior to the study. Anesthesia was induced by an intramuscular injection of $0.05 \mathrm{~mL} / \mathrm{kg}$ body weight $(\mathrm{BW})$ of a mixture of telazole, xylazine, and ketamine, all at a concentration of $100 \mathrm{mg} / \mathrm{mL}$. Following endotracheal intubation, the swine were ventilated with an approximate fraction of inspired oxygen $\left(\mathrm{FiO}_{2}\right)$ of 0.6 to avoid hypoxemia and anesthesia was maintained with 1.0 to $2.5 \%$ isoflurane. A 21-gauge catheter was inserted in an ear vein and a mixture of dextrose $5 \%$ and $\mathrm{NaCl} 0.9 \%$ (Baxter, Deerfield, IL, USA) was infused at $1 \mathrm{~mL} / \mathrm{kg} /$ hour thereafter.

A $7 \mathrm{Fr}$ introducer was placed in the right internal jugular vein and a continuous cardiac output (CCO) pulmonary artery catheter equipped with fiber optics (Vigilance catheter, Edwards Lifesciences, Irvine, CA, USA) was floated to measure $\mathrm{CCO}$ and $\mathrm{SvO}_{2}$. A triple- 
lumen 18-gauge catheter for blood sampling and blood pressure monitoring using a low-volume pressure transducer (MP50, Gould Inc., Cleveland, OH, USA) was inserted in the right femoral artery, as well as an $8 \mathrm{Fr}$ introducer in the right femoral vein. A urine catheter was surgically inserted in the bladder through a suprapubic incision.

Three microdialysis catheters (30 mm long, $100 \mathrm{kDa}$ molecular weight cutoff membranes) (CMA 71, M Dialysis $A B$, Stockholm, Sweden) were inserted in the left gluteus maximus muscle, between the loops of small intestine through the suprapubic incision, and in the right lobe of the liver (right anterior medial segment V) through a 4$\mathrm{cm}$ midline incision starting at the level of the xiphoid process. The microdialysis catheters in liver and muscle were placed using a splitable needle (M Dialysis $A B$, Stockholm, Sweden) as previously described [19].

\section{Hemorrhage and resuscitation protocol}

We used a modified version of a pressure-based hemorrhagic shock and resuscitation protocol previously described by us [20] (Figure 1). Briefly, after surgery swine were allowed to stabilize for 30 minutes. Using a roller pump (Masterflex ${ }^{\mathrm{Tw}} \mathrm{L} / \mathrm{S}$-Easy-load II, Barnant Co., Barrington, IL, USA) swine were then bled at $20 \mathrm{~mL} / \mathrm{min}$ through the femoral artery until mean arterial pressure (MAP) had decreased to $30 \mathrm{mmHg}$. Repeated episodes of bleeding (at $60 \mathrm{ml} / \mathrm{min}$ ) were done if the animal was able to spontaneously recover to a MAP of $40 \mathrm{mmHg}$, thus maintaining the animal between 30 and $40 \mathrm{mmHg}$ for a

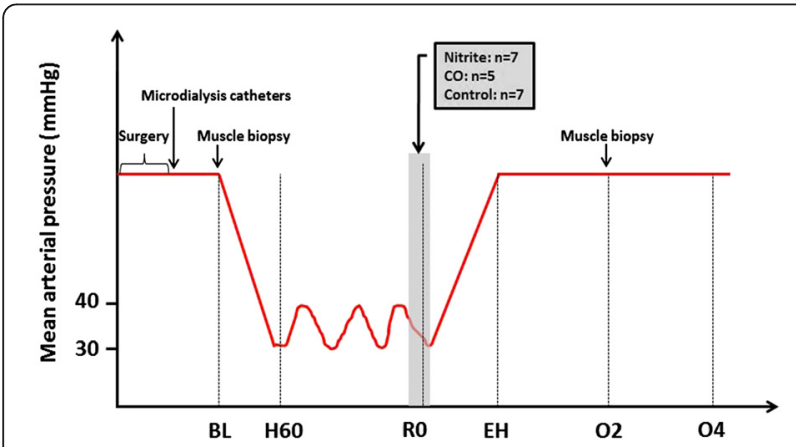

Figure 1 Study protocol and experimental design. In the first bleeding period (baseline $(\mathrm{BL})$ to hemorrhage $60(\mathrm{H} 60)$ the animals were bled at a rate of $20 \mathrm{~mL} / \mathrm{min}$. They were then kept at mean arterial pressures (MAP) between 30 and $40 \mathrm{mmHg}$ for 90 minutes (H60 to start resuscitation (R0) by repeatedly hemorrhages if necessary at rates of $60 \mathrm{~mL} / \mathrm{min}$. At the end of this period the animals were administered the study drug (nitrite or CO). RO samples were obtained before administration of study drug (shadow). The animals were resuscitated with Hextend ${ }^{\mathrm{TM}}$ at a volume equal to the total bleeding amount at a rate of $60 \mathrm{~mL} / \mathrm{min}$. They were then kept at MAP equal to or above their baseline MAP using norepinephrine, and mixed venous oxygen saturation $\left(\mathrm{SvO}_{2}\right)>70 \%$ using dobutamine throughout a fourhour observation period until scheduled euthanasia. total of 90 minutes, defined as the shock period. Resuscitation was started either when the 90-minute shock period had elapsed, or when the swine had evidence of cardiovascular collapse defined as MAP below $30 \mathrm{mmHg}$ for $5 \mathrm{mi}-$ nutes or below $20 \mathrm{mmHg}$ for 10 seconds. Resuscitation was done with Hextend ${ }^{\mathrm{mi}}$ (6\% hetastarch with electrolytes, glucose $99 \mathrm{mg} / \mathrm{dL}$, and lactate $28 \mathrm{mM}$ (Hospira Inc., Lake Forest, IL, USA) at equal parts as shed blood, at a rate of $60 \mathrm{~mL} / \mathrm{min}$. After this initial bolus, the animals could receive crystalloids (Ringer's lactate) and/or vasopressors to maintain baseline MAP according to a prespecified algorithm described elsewhere [20]. Animals were then observed for a 4-hour period that started at the end of Hextend $^{\mathrm{Tx}}$ infusion and finalized with scheduled euthanasia.

\section{Measurements in microdialysate and blood}

The microdialysis catheters were perfused with dextran 60 and electrolytes (Plasmodex ${ }^{\mathrm{Tn}}$, Meda AB, Stockholm, Sweden) at $1 \mu \mathrm{l} / \mathrm{min}^{-1}$ by microinjection pumps (CMA 107, M Dialysis AB, Stockholm, Sweden), as previously described by us [19]. Starting at the end of the 30minute stabilization period samples were collected every 30 minutes and analyzed for glucose, lactate, pyruvate, and glycerol using a microdialysis analyzer (Iscus, $\mathrm{M}$ Dialysis AB, Stockholm, Sweden). The samples obtained after the hemorrhage period ( $\mathrm{H} 60)$ were collected prior to starting nitrite or $\mathrm{CO}$ in those two animal groups. After finishing the analyses the samples were frozen in liquid nitrogen and transferred to the $-80^{\circ} \mathrm{C}$ freezer.

Simultaneously, arterial and mixed venous blood samples were taken and analyzed in a blood gas analyzer (ABL-90 ${ }^{\mathrm{m}}$, Radiometer, Copenhagen, Denmark) for $\mathrm{pH}$, $\mathrm{pCO}_{2}, \mathrm{pO}_{2}, \mathrm{SO}_{2}$, hemoglobin, carboxyhemoglobin $(\mathrm{COHb})$, methemoglobin (MetHb), glucose and lactate.

Stored plasma and microdialysate samples were subsequently analyzed as described elsewhere [21] for concentrations of nitrite by injecting plasma samples into a glass chamber containing tri-iodide, which generated NO, which was subsequently measured by ozone chemiluminescence using a Sievers 280i nitric oxide analyzer according to the instructions of the manufacturer (General Electric, Boulder, CO, USA). Plasma samples were also analyzed for aspartate aminotransferase (AST), alanine aminotransferase (ALT), and creatinine (Sigma-Aldrich assay kit, St. Louis, MO, USA).

\section{Mitochondrial function}

Biopsies from the left rectus femoris muscle ventral of the microdialysis catheter were taken at the end of the stabilization period (baseline) and two hours after end of fluid resuscitation (Figure 1). These samples were immediately homogenized and measured after collection. Oxygen consumption in the muscle biopsies were immediately measured using a Clark-type oxygen electrode (Instech 
Laboratories, Plymouth Meeting, PA, USA) in the presence of succinate (for State 4 measurements) and adenosine diphosphate (ADP) (for State 3 measurement). Respiratory control ratio (RCR) was calculated as State 3/ State 4.

Complex I activity: complex I activity was measured spectrophotometrically in biopsied tissue by spectrophotometrically monitoring the oxidation of nicotinamide adenine dinucleotide dehydrogenase (NADH) in the presence of coenzyme $\mathrm{Q}$ and the presence and absence of rotenone.

Aconitase activity: the enzymatic activity of aconitase was measured in lysed biopsies by spectrophotometrically monitoring the formation of nicotinamide adenine dinucleotide phosphate (NADPH) at $340 \mathrm{~nm}$ using the Bioxytech Aconitase-340 kit (Oxis Research, Foster City, CA, USA).

Carbonyl measurement: carbonyl levels were assessed in homogenized tissue spectrophotometrically $(285 \mathrm{~nm})$ after derivitization of the carbonyls with 2-dinitrophenylhydrazine as per the Colorimetric Carbonyl kit (Cayman Chemicals, Ann Arbor, MI, USA).

\section{Experimental protocol}

Animals were randomized to one of the three prespecified groups at the end of first bleed: nitrite $(n=7,30$ minute nebulization of sodium nitrite at a dose of $11 \mathrm{mg}$ sodium nitrite in $2.5 \mathrm{~mL}$ phosphate-buffered saline independent of $\mathrm{BW}) ; \mathrm{CO}(\mathrm{n}=5,30$-minute inhalation of $\mathrm{CO}$ at $250 \mathrm{ppm}$ independent of $\mathrm{BW})$; or control $(\mathrm{n}=7$, no further intervention) (Figure 1). Microdialysis monitoring was implemented in the study protocol in the last 20 in a series of 66 pigs in total, explaining the uneven distribution of animals in each group. So there was no bias as to groups, but differences in group totals. The nitrite dose is on the low end of what was previously demonstrate to be effective in order to minimize vasodilation in the setting of shock [16]. Either nitrite or $\mathrm{CO}$ was started 30 minutes prior to initiation of resuscitation, 60 minutes into the ischemic shock period and continued until the end of resuscitation. One animal served as sham, by undergoing the same surgical procedure but without bleeding or administration of nitrite or $\mathrm{CO}$. Additionally, we present complex I and acotinase activity and carbonyl levels from one sham animal that was included before microdialysis catheters were implemented in the protocol.

\section{Statistical analyses}

Between-group comparisons of macrohemodynamic and systemic oxygenation parameters were performed with the Kruskal-Wallis and Mann-Whitney $U$ tests. The changes in mitochondrial RCR values from baseline to the two-hour observation point were analyzed with the Wilcoxon signed-rank test and group differences were explored with the Mann- Whitney $U$ test. Complex 1 and aconitase activities and levels of carbonyl were compared to controls with the Mann-Whitney $U$ test. Values for AST, ALT, and creatinine at baseline and at the fourhour observation time point were analyzed with the Wilcoxon signed-rank test. Other repeated measures were analyzed in a random effects mixed model: effects of different animals, groups, BW, and amount of bleeding were included in the model aiming at achieving low information criteria. Since the data were not normally distributed the mixed model analyses were performed on $\log 10$-transformed data. Estimated fixed effects (e.f.e.) of factors and covariates are reported; values $>1$ represent positive effects and values $<1$ represent negative effects. The $P$ values are two-tailed and Bonferroni-adjusted for comparison of three groups. $P$ values lower than 0.05 were considered to indicate statistical significance (PASW 21.0, IBM Corp. Armonk, NY, USA).

\section{Results}

Seven animals were randomized to the control and nitrite groups and five to the $\mathrm{CO}$ group. The pigs in the control group had significantly lower BW than the animals in the remaining two groups despite randomization (Table 1). All animals appeared healthy upon arrival and tolerated the surgical procedure, including insertion of microdialysis catheters, well and bleeding and resuscitation phases of the protocol were carried out without untoward complications. However, as expected based on the severity of the hemorrhagic shock protocol, three animals developed severe hypotension and died 49 (control), 51 (nitrite), and 88 (nitrite) minutes after starting resuscitation. Data from these animals were not excluded from analyses. The remaining animals were observed for a median of 253 minutes (range 230 to 260 minutes) and completed the protocol.

\section{Macrohemodynamic and systemic oxygenation parameters}

Hemodynamic behavior of animals followed expected trends regardless of the groups they were allocated to as summarized in Figure 2. Pharmacological preconditioning did not have any effect on MAP, stroke volume variation (SVV), heart rate (HR), CCO, or mean pulmonary artery pressure (MPAP) when compared to control animals. The MAP target of $30 \mathrm{mmHg}$ (time point $\mathrm{H} 0$ ) was reached after a median of 33 minutes (range 23 to 55 minutes), with no time difference between groups $(P=0.20)$. Resuscitation was triggered by the 90 -minute shock period criteria at R0 in all animals but one, in which resuscitation was started 13 minutes into the shock period for cardiovascular collapse (defined previously). All but one animal (control) required norepinephrine to keep their MAP at baseline values following resuscitation and the 
Table 1 Body weight, surgery time, and hemorrhagic and resuscitation data in 19 pigs randomized to inhale nitrite, carbon monoxide, or no inhalation (control)

\begin{tabular}{|c|c|c|c|c|c|c|c|c|}
\hline & \multicolumn{2}{|c|}{ Control $(n=7)$} & \multicolumn{3}{|c|}{ Nitrite $(n=7)$} & \multicolumn{3}{|c|}{ Carbon monoxide $(n=5)$} \\
\hline & Median & Range & Median & Range & $P$ value $^{1}$ & Median & Range & $P$ value ${ }^{1}$ \\
\hline Body weight (kg) & 29.5 & $27.2-31.4$ & 33.7 & $30.4-36.4$ & 0.004 & 31.1 & $28.0-35.8$ & 0.40 \\
\hline Surgery time (minutes) & 70 & $61-75$ & 75 & $64-87$ & 0.26 & 78 & $71-90$ & 0.10 \\
\hline \multicolumn{9}{|l|}{ Shed blood volume } \\
\hline $\mathrm{mL}$ & 1000 & $500-1241$ & 1025 & $761-1452$ & $>0.99$ & 711 & $652-1138$ & 0.54 \\
\hline$\%$ of $\mathrm{TBV}^{2}$ & 51 & $27-70$ & 46 & $38-63$ & $>0.99$ & 36 & $29-56$ & 0.40 \\
\hline \multicolumn{9}{|l|}{ Duration (minutes) } \\
\hline Hemorrhage $1^{3}$ & 37 & $28-55$ & 34 & $26-42$ & 0.77 & 28 & $23-33$ & 0.06 \\
\hline Resuscitation & 15 & $8-20$ & 16 & $12-23$ & $>0.99$ & 11 & $8-23$ & $>0.99$ \\
\hline
\end{tabular}

${ }^{1}$ Nitrite and carbon monoxide groups were compared with the control group by the Mann-Whitney $U$ test. The $P$ values were Bonferroni-adjusted for comparison of three groups; ${ }^{2} \mathrm{TBV}$ : total blood volume based on an assumed blood volume of $65 \mathrm{~mL} / \mathrm{kg}$; ${ }^{3}$ hemorrhage 1 : time until mean arterial pressure was lower than $30 \mathrm{mmHg}$.

median maximum norepinephrine infusion rate was $0.15 \mu \mathrm{g} / \mathrm{kg} / \mathrm{min}$ (range 0 to $0.4 \mu \mathrm{g} / \mathrm{kg} / \mathrm{min}$ ). There was no difference in the norepinephrine infusion rate between groups $(P=0.77)$. None of the animals required dobutamine.
$\mathrm{SvO}_{2}$ mirrored $\mathrm{CCO}$ and MAP changes, and hemoglobin dropped during resuscitation, as expected (Figure 2). There were no significant group differences in $\mathrm{SvO}_{2}$ or hemoglobin values between groups during hemorrhage or resuscitation.

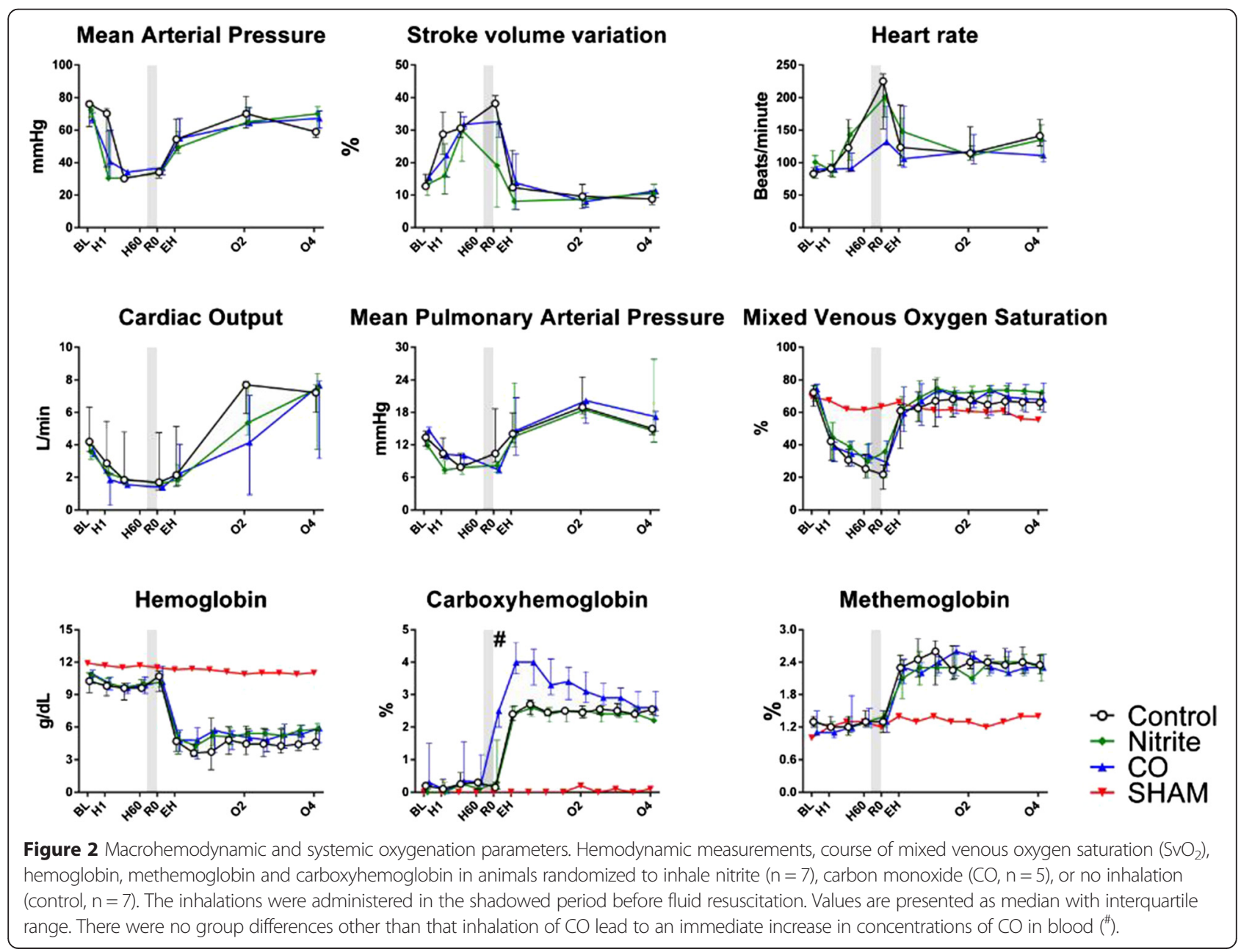




\section{Toxicity hemoglobin parameters}

We found a marked elevation of MetHb after resuscitation in all animals (e.f.e. of going from period RO to EH 1.21 (95\% CI (Confidence interval) 1.18 to $1.24, P$ $<0.001)$, independent of group $(P=0.84)$. High BW was slightly negatively correlated with MetHb (e.f.e. 0.99 (95\% CI 0.98 to 1.00) $(P=0.05)$ ), whereas amount of shed blood had no significant impact $(P=0.38)$ (Figure 2). Administration of $\mathrm{CO}$ led to an immediate increase of $\mathrm{COHb}$ (e.f.e. of going from $\mathrm{H} 60$ to $\mathrm{RO} 1.26$ (95\% CI 1.00 to $1.52, P=0.001)$, independent of weight $(P=0.15)$ or amount of shed blood $(P=0.99)$. Resuscitation led to an increase of $\mathrm{CO}$ in all animals (e.f.e. of going from period RO to EH 1.52 (95\% CI 1.41 to $1.64, P$ $<0.001$ ), and there was no difference between the nitrite and control group $(P>0.99)$. The highest measured $\mathrm{COHb}$ in the $\mathrm{CO}$ group was $4.6 \%$ [22].

Microdialysis metabolic parameter trends:

1. Nitrite (Figure 3). During hemorrhage nitrite was unchanged in plasma, muscle, and peritoneal fluid and liver. Following inhalation of nebulized sodium nitrite prior to fluid resuscitation there was a significant increase in nitrite in plasma (e.f.e. of R0 compared to $\mathrm{H} 60$ to was 1.51 (95\% CI 1.17 to 1.85 , $P=0.01)$ ). Fluid resuscitation did not lead to further increase in plasma nitrite, and until the end of the observation period, plasma nitrite decreased with an hourly e.f.e. of 0.91 (95\% CI 0.86 to 0.96$)(P=0.003)$.
As opposed to the nitrite group, fluid resuscitation implied an increase of plasma nitrite both in the control group and $\mathrm{CO}$ group; e.f.e. of $\mathrm{EH}$ compared to R0 in the control group was 1.16 (95\% CI 1.01 to 1.32$)(P=0.04)$, and 1.22 (95\% CI 1.00 to 1.43$)$ $(P=0.05)$ in the CO group. Also different from the nitrite group, the nitrite levels did not decrease until the end of the study period neither in the control group $(P=0.90)$ nor in the $\mathrm{CO}$ group $(P=0.09)$. Following nitrite inhalation, concentrations of nitrite did not increase in microdialysis samples neither from muscle $(P=0.74)$ nor from peritoneal fluid $(P=0.44)$ (no samples were collected at the H60 observation time point in liver). Resuscitation did not influence nitrite concentrations in any of the microdialysis samples $(P>0.05$ for all). As opposed to the time-dependent decrease in plasma nitrite in the nitrite group after fluid resuscitation, no changes were observed in any of the microdialysis samples in the nitrite group ( $P>0.05$ for all). In the $\mathrm{CO}$ group, the nitrite concentrations increased time-dependently in all microdialysis samples $(P<0.05$ for all). Similar changes were found in peritoneal fluid and liver in the control group ( $P<0.05$ for both), whereas the increase in muscle nitrite was not significant $(P=0.12)$. The pig's weight and amount of bleeding did not have any statistically significant influence in any of the performed statistical analyses.
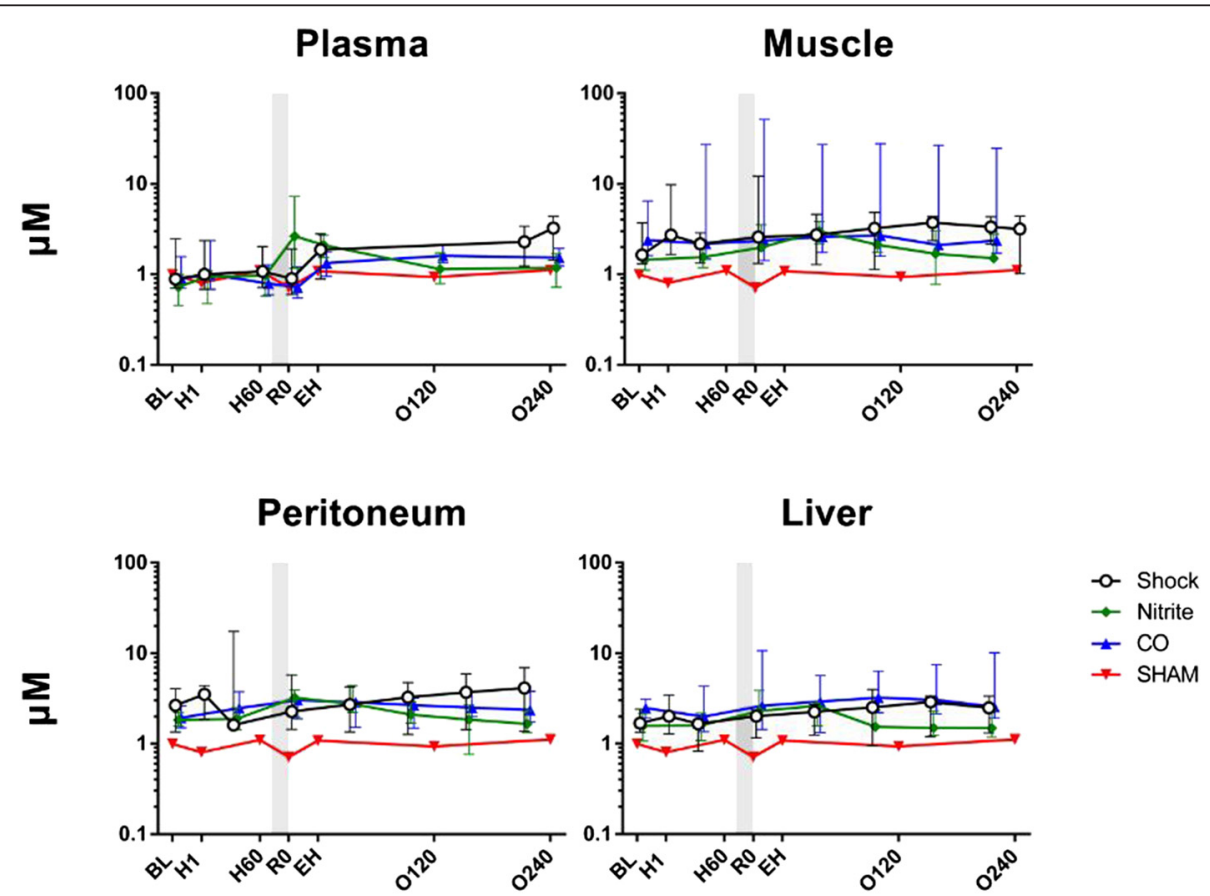

Figure 3 Nitrite concentrations. Nitrite values over time for nitrite $(n=7)$, carbon monoxide $(C O, n=5)$, or control $(n=7)$ groups. One animal was not bled (sham). The nitrite and CO inhalations timing is displayed by the shadow prior to resuscitation. Values are presented as median with interquartile range. 
2. Lactate (Figure 4). During hemorrhage and until fluid resuscitation lactate increased at all measured sites. The steepest increase was seen in peritoneal fluid with an hourly e.f.e. of 1.26 (95\% CI 1.23 to 1.29), followed by blood (1.20 (95\% CI 1.17 to 1.22 ), liver 1.14 (95\% CI 1.10 to 1.18), and muscle 1.07 (95\% CI 1.05 to 1.10$)(P<0.001$ for all). There were no group differences. High amount of bleeding was positively associated with lactate in peritoneal fluid (e.f.e. of each dL hemorrhage was 1.04 (95\% CI 1.02 to 1.07$)(P=0.02)$. Following resuscitation lactate continued to increase. Inhalation of nitrite had a negative effect on this continued increase in blood; the e.f.e. on lactate in arterial blood was 0.74 (95\% CI 0.52 to 0.95$)(P=0.04)$. After the post-resuscitation peak lactate decreased time dependently $(P<0.001$ for all sites), and there were no group differences.
3. Pyruvate. In general, the course of pyruvate concentrations throughout the experiments mirrored the lactate concentrations. As opposed to lactate, the increases during hemorrhage were not as steep as for lactate, and amount of hemorrhage had no influence on the values. There was a nonsignificant negative effect of inhaling nitrite and $\mathrm{CO}$ prior to fluid resuscitation.

4. Lactate to pyruvate ratio (LPR) (Figure 4). During hemorrhage, there was a time-dependent increase in LPR only in peritoneal fluid and the hourly e.f.e. was 1.14 (95\% CI 1.12 to 1.16$)(P<0.001)$. None of the inhaled agents had any effect on the post-reperfusion LPR values, and there were no group differences in the post-reperfusion period. Following reperfusion, no group differences were found for LPR in any of the tissues

\section{A Lactate}
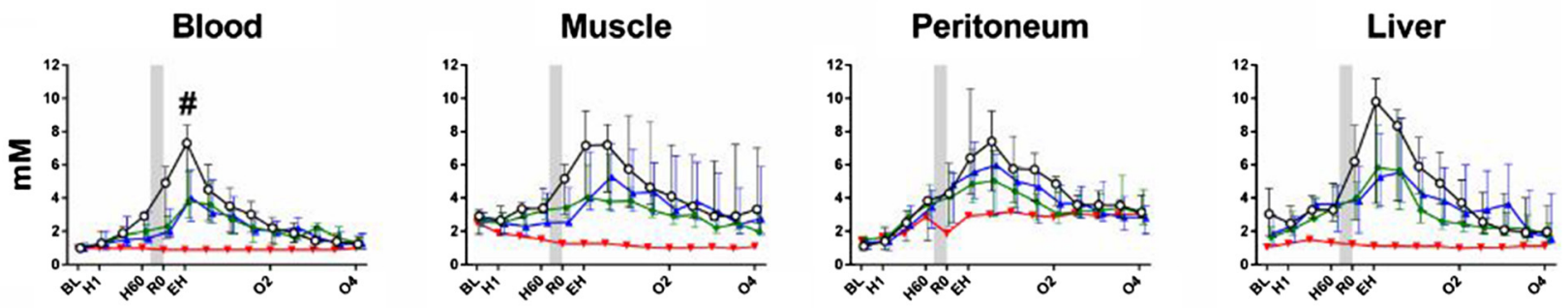

\section{B Lactate/ pyruvate}
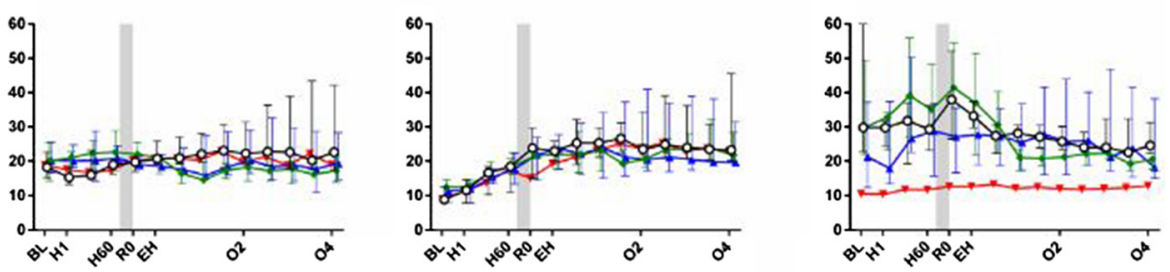

\section{Glycerol}
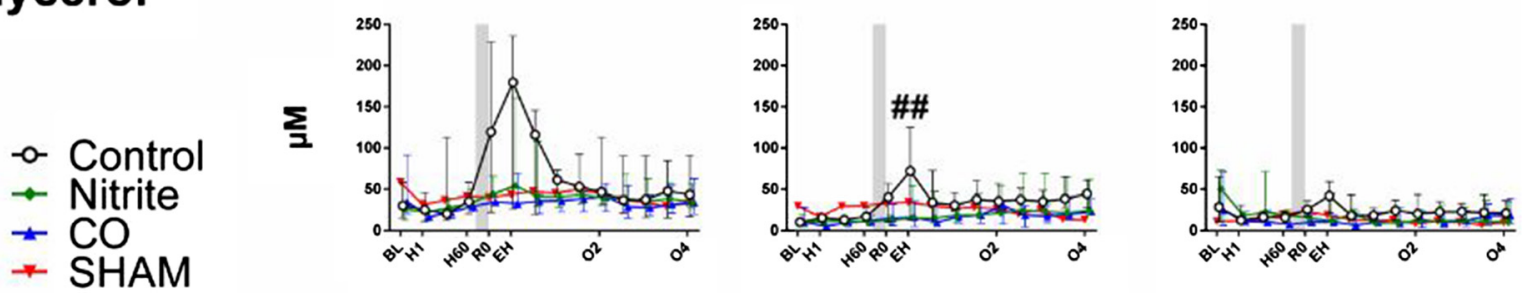

Figure 4 Metabolic parameters. Lactate (A), lactate/pyruvate ratio (B), and glycerol (C) values over time for nitrite $(\mathrm{n}=7)$, carbon monoxide (CO, $n=5)$, or control $(n=7)$ groups. One animal was not bled (sham). The nitrite and CO inhalations timing is displayed by the shadow prior to resuscitation. Values are presented as median with interquartile range. ${ }^{\#} P<0.05$ for the nitrite group compared to controls. ${ }^{\#} P<0.05$ for nitrite and $\mathrm{CO}$ groups compared to controls. 
5. Glucose. During hemorrhage, there was a time-dependent increase in the glucose concentrations in blood with an hourly e.f.e. of 1.04 (95\% CI 1.02 to 1.06$)(P<0.001)$. As opposed to blood, there were time-dependent decreases in the glucose concentrations in peritoneal fluid (e.f.e. 0.88 (95\% CI 0.82 to 0.94$)(P<0.001))$, liver (e.f.e. 0.89 (95\% CI 0.83 to 0.95$)(P<0.001)$ ), and muscle (e.f.e. 0.91 (95\% CI 0.87 to 0.96$)(P=0.001))$. Following fluid resuscitation the concentrations of glucose in tissues tended to increase, but only reaching statistically significance in peritoneal fluid with an e.f.e. of resuscitation of 1.12 (95\% CI 1.00 to 1.23 ) $(P=0.04)$, without altering blood glucose (e.f.e. 1.00 (95\% CI 0.96 to 1.04$)(P=0.87)$. Thereafter, and until the end of the observation period, glucose increased at all measured sites $(P<0.001$ for all). There was no effect of any of the inhaled agents on the post-reperfusion values.

6. Glycerol (Figure 4). During hemorrhage there was a time-dependent increase in the cell damage marker glycerol in muscle (hourly e.f.e. of 1.15 (95\% CI 1.09 to 1.22$)(P<0.001)$ and peritoneal fluid (hourly e.f.e. of 1.09 (95\% CI 1.03 to 1.15$)(P=0.006)$ ). Amount of bleeding was positively correlated with glycerol values ( $P<0.05$ for both), and weight did not have any influence. Statistical analyses were not performed for the hemorrhage period in liver since we assume that the high initial values for glycerol were related to tissue damage caused by needle insertion. Inhalation of nitrite implied reduced release of glycerol to peritoneal fluid as compared to controls and the e.f.e. of nitrite was 0.41 (95\% CI 0.10 to 0.73$)(P=0.002)$. The same tendency was observed for $\mathrm{CO}$ (e.f.e. 0.68 (95\% CI 0.59 to 0.96$)(P=0.06))$. Thereafter, and until the end of the study period, the values normalized and there were no group differences.

\section{Markers of organ damage}

Plasma AST and creatinine increased significantly from samples collected at the end of the baseline period to the four-hour time point. AST increased from median $29 \mathrm{U} / \mathrm{L}$ (range 17 to $43 \mathrm{U} / \mathrm{L}$ ) to $70 \mathrm{U} / \mathrm{L}$ (range 17 to 102
$\mathrm{U} / \mathrm{L})(P<0.001)$ and creatinine increased from median $1.0 \mathrm{mg} / \mathrm{dL}$ (range 0.2 to $1.4 \mathrm{mg} / \mathrm{dL}$ ) to $1.3 \mathrm{mg} / \mathrm{dL}(0.2$ to $1.8 \mathrm{mg} / \mathrm{dL})(P=0.001)$. As opposed to AST and creatinine, the ALT values decreased slightly from median 41 $\mathrm{U} / \mathrm{L}$ (range 2 to $67 \mathrm{U} / \mathrm{L}$ ) to $34 \mathrm{U} / \mathrm{L}$ (range 17 to $54 \mathrm{U} / \mathrm{L}$ ). There were no group differences at any of the time points (Table 2).

\section{Mitochondrial function}

Comparison of the RCR two hours after fluid resuscitation to the RCR in the same animal at baseline showed that RCR was significantly decreased in the control group over the course of shock and resuscitation $(P=0.04)$, but unchanged in both the nitrite $(P=0.45)$ and $C O(P>0.99)$ groups (Figure 5). The magnitude of decrease in RCR observed in the control group was significantly greater than in the nitrite group $(P=0.05)$, but not compared to the CO group $(P=0.30)$. The decrease in RCR over the time course of the experiment was driven both by an increase in State 4 respiration and a decrease in State 3 in the control group, indicative of increased proton leak and decreased oxidative phosphorylation capacity respectively (Figure 5B-C).

\section{Antioxidant mechanisms}

Both CO and nitrite have been shown to mediate antioxidant effects, particularly in hypoxic models [14-16]. Measurement of protein carbonyls as a marker of protein oxidation showed a significant decrease with nitrite $(P=0.004)$ and CO $(P=0.002)$ treatment compared to control animals (Figure 6A). As a second measure of oxidative stress, the activity of aconitase (an iron-sulfur enzyme particularly susceptible to oxidative inactivation) was measured. Though not significant, both nitrite $(P=0.15)$ and $\mathrm{CO}(P=0.08)$ treatment showed a strong trend toward preserving aconitase activity (Figure 6B), consistent with the prevention of oxidative stress. Further, nitrite has been shown to inhibit complex I activity to attenuate mitochondrial ROS generation. Measurement of complex I activity showed nitrite-dependent inhibition $(P=0.02)$, but no change by $\mathrm{CO}(P=0.52)$ (Figure 6C).

Table 2 Circulating markers of organ damage measured at end of baseline and at the end of the study

\begin{tabular}{|c|c|c|c|c|c|c|c|c|}
\hline & \multicolumn{4}{|l|}{ Baseline } & \multicolumn{4}{|c|}{ Four hours after completed resuscitation } \\
\hline & Control $(n=7)$ & Nitrite $(n=7)$ & $C O^{*}(n=5)$ & $P$ value** & Control $(n=6)$ & Nitrite $(n=5)$ & $\mathrm{CO}^{*}(\mathrm{n}=5)$ & $P$ value ${ }^{* *}$ \\
\hline $\begin{array}{l}\text { Aspartate aminotransferase } \\
(\mathrm{U} / \mathrm{L})\end{array}$ & $27(17-34)$ & $30(20-41)$ & $34(18-43)$ & 0.96 & $68(63-99)$ & $66(46-102)$ & $73(39-87)$ & $>0.99$ \\
\hline $\begin{array}{l}\text { Alanine aminotransferase } \\
(\mathrm{U} / \mathrm{L})\end{array}$ & $36(21-52)$ & $41(28-67)$ & $45(28-47)$ & $>0.99$ & $37(18-54)$ & $26(17-53)$ & $35(20-44)$ & $>0.99$ \\
\hline Creatinine (mg/dL) & $1.0(0.2-1.4)$ & $1.0(0.8-1.2)$ & $1.2(0.7-1.4)$ & 0.83 & $1.4(1.1-1.5)$ & $1.2(1.1-1.3)$ & $1.3(1.1-1.8)$ & 0.37 \\
\hline
\end{tabular}

*CO, carbon monoxide; ${ }^{* *}$ group differences were explored with the Kruskal-Wallis test and the $P$ values were Bonferroni-adjusted for comparisons of three groups. 


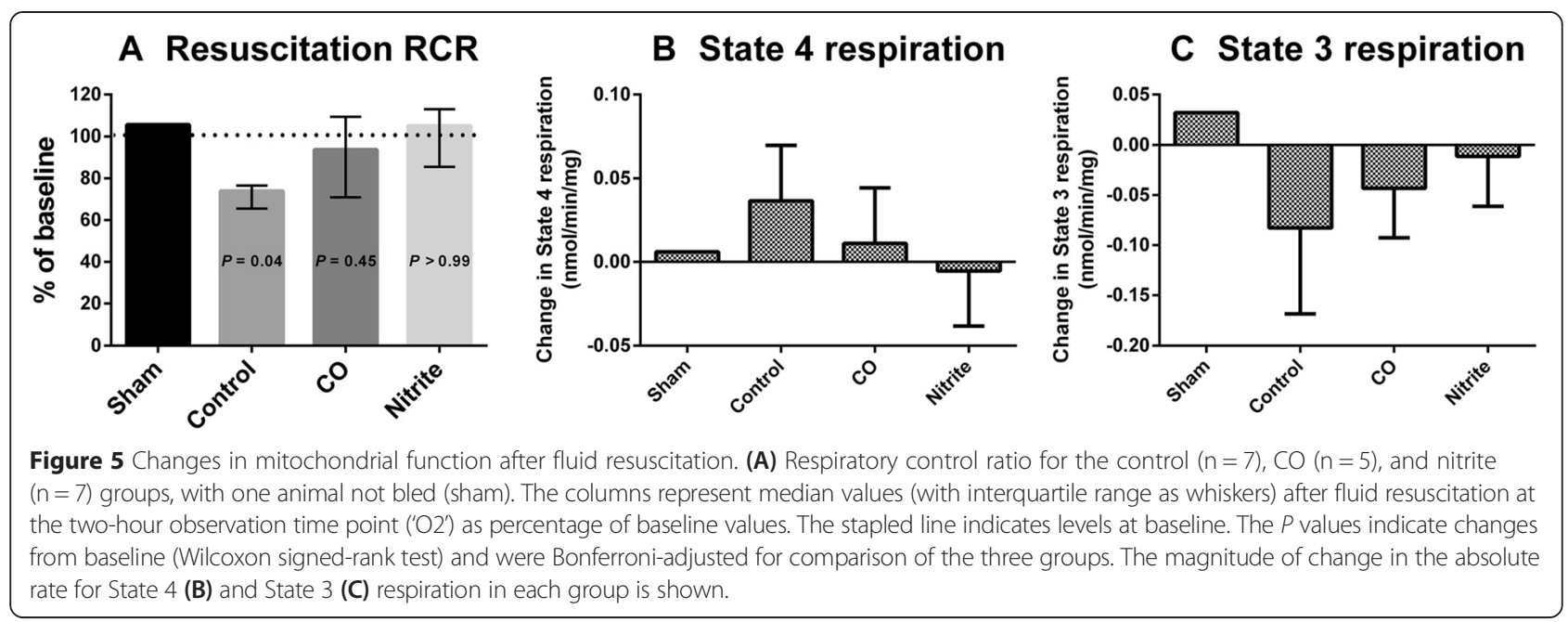

\section{Discussion}

This study suggests that in the setting of severe hemorrhagic shock, the administration of low-dose nebulized $\mathrm{NaNO}_{2}$ or inhaled $\mathrm{CO}$ may be able to reduce the short-term ischemic and necrotic effects of reperfusion without altering macrohemodynamic and systemic oxygenation parameters although reduced concentrations of lactate were only found in blood and reduced concentrations of glycerol were only found in peritoneal fluid $[19,23,24]$. In accordance to this, these data also demonstrates that $\mathrm{NaNO}_{2}$ or $\mathrm{CO}$, but not standard resuscitation alone, maintain coupling of the electron transport chain (assessed with the respiratory controlled ratio) and prevented mitochondrial dysfunction. Collectively, these findings are consistent with the hypothesis that inhaled $\mathrm{NaNO}_{2}$ and $\mathrm{CO}$ may limit reperfusion injury when used as adjuncts to standard resuscitation.

The mechanisms by which $\mathrm{NaNO}_{2}$ and $\mathrm{CO}$ exert protective effects are still unknown, although available data have consistently suggested that their main action may be at the mitochondrial level [14]. Nitrite has been shown to decrease apoptosis and cell damage in other models such as ischemia reperfusion, and across different organs, such as the heart, liver, brain and kidney [25-32]. Fast reactivation of complex I during reperfusion is recognized as a central event in ischemia/reperfusion-induced cell injury as it is related with increased production of ROS [5]. It follows that blockade of this reactivation lends a robust protective mechanism [11-13]. Recent data have suggested that nitrite may exert its protective effects through S-nitrosation of complex I of the respiratory chain. Chouchani et al. were able to demonstrate that nitrite selectively and reversibly S-nitrosates Cys 39 on the ND3 subunit of mitochondrial complex I [33]. This inhibition is associated with a marked reduction in mitochondriaderived ROS production, and also with reduced cell damage, necrosis and apoptosis. Our data supports these prior findings, as the respiratory control ratio, a measure of coupling of the electron transport chain, was higher in the nitrite group as compared to control.

As expected, administration of $\mathrm{NaNO}_{2}$ resulted in increased circulating and tissue nitrite levels. However, as shown in Figure 3, after an initial peak we found a tendency toward declining nitrite levels in our nitrite-treated

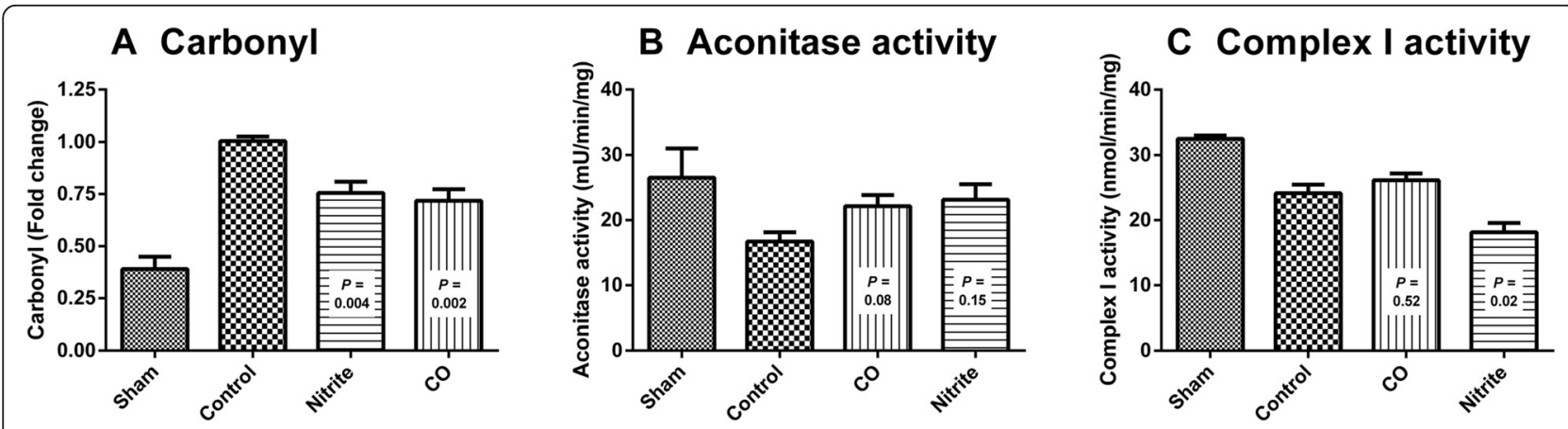

Figure 6 Carbonyl levels and aconitase- and complex I activity. Columns represent means +/- standard error of the mean (SEM) of (A) carbonyl levels, (B) aconitase activity and (C) complex I activity in sham $(n=2)$, control $(n=7)$, nitrite $(n=7)$ and carbon monoxide $(C O)(n=5)$ treated animals. The $P$ values indicate comparison of treated groups to controls (Mann-Whitney $U$ test) and were Bonferroni-adjusted for comparison of the three groups. 
group, as opposed to the control group where post-resuscitation nitrite concentrations showed an increasing tendency. In the body, $\mathrm{NO}_{2}^{-}$is reduced to $\mathrm{NO}$ by different mechanisms including enzymatic reduction by hemoglobin and myoglobin, interaction with components of the electron transport chain, and the xanthine oxidoreductase system [16,34]. Importantly, hypoxia and cell injury promote conversion of $\mathrm{NO}_{2}^{-}$to $\mathrm{NO}$. We speculate that nitrite-induced decreases in ROS production resulted in less vascular endothelial stress reducing the normal response to this stress, which is NO production by endothelial NO synthase (eNOS). Since nitrite is the one electron oxidation product of $\mathrm{NO}$ and an excellent measure of eNOS activity [35], we presume the lower nitrite levels reflect lower eNOS activation. Another possibility would be that animals exposed to nitrite could have a higher rate of conversion to NO and thus, present with lower systemic levels. This does not seem to be the case as these animals had similar MAP, CCO, blood lactate, and $\mathrm{SvO}_{2}$ to the other groups.

Carbon monoxide blocks cytochrome c oxidase complex (or complex IV) in the mitochondrial electron transport chain, which is associated with a low level, but still increased production of ROS upstream at complex III. Acting as second messengers for $\mathrm{CO}$, these ROS trigger adaptive responses and result in protection of the cell and tissues [15,36,37]. One adaptive response is the activation of the master energy regulator, adenosine monophosphateactivated protein kinase (AMPK), and subsequent stimulation of mitophagy by which dysfunctional mitochondria are self-digested in autophagosomes and their components recycled as energy substrates [38,39].

The microdialysis method is a useful way to perform repeated measurements of small molecules in the extracellular space. As such, it is a useful tool to investigate effects of preconditioning agents at the tissue or organ level [18]. Glycerol forming the backbone of the fatty acids in the cell membrane is one of these important small molecules. It is released under ischemic conditions by activation of phospholipase A2 and it is an evident marker of cell injury $[19,23,40]$. In the present study, the most consistent effects of pharmacologic preconditioning, as judged by lower glycerol values, were found in the peritoneal fluid, suggesting that both $\mathrm{NaNO}_{2}$ and $\mathrm{CO}$ may protect the intestine from injury caused by ischemia and subsequent reperfusion $[41,42]$. Since the gut mucosa is at a higher risk for ischemia/reperfusion injury than other tissues, like muscle and liver, these finding suggest a potential therapeutic role of these inhalational agents. Similar results were found in a study investigating the effects of ischemia preconditioning of heart muscle prior to coronary artery occlusion [43]. In a recent study, remote ischemic preconditioning led to reduced levels of glycerol in ischemic brain tissue [44], and similar effects of direct ischemic preconditioning have been found in liver tissue $[45,46]$. Furthermore, preconditioning has been shown to sustain lactate, pyruvate and the LPR in liver, muscle, free flaps and heart [46-49].

\section{Study limitations}

Although we demonstrated effects of preconditioning agents in the extracellular space, we had only small deteriorations in organ function as measured by AST, but a $30 \%$ increase in creatinine four hours post shock. Thus, the detrimental effect of this severe hemorrhagic shock model may be dissimilar among organs and tissues. Furthermore, we did not find any group differences in AST or creatinine increases. In the setting of hemorrhagic shock, organ injury and dysfunction may not become readily apparent in the acute phase, and manifest only later following resuscitation. We reason that this was why we did not see greater evidence of organ dysfunction. Potentially, studies that include a longer window of observation may provide further insight into whether these effects on mitochondrial function will translate into protecting organ function. In addition, despite using randomization, total BW was different in the three groups, which can largely affect our results since bleeding rate was at $20 \mathrm{~mL} / \mathrm{min}$ independent of BW. However, the effect of $\mathrm{BW}$ on the measured variables was small and furthermore, was controlled for in the statistical model. Importantly, BW had no effect on the concentrations of glycerol. A larger sham group allowing statistical comparisons would have improved the methodical strength of the study. Finally, we did not allow for any stabilization after needle and microdialysis catheter insertion in muscle and liver before starting microdialysate sampling, and thus the high baseline values in some animals most likely reflect local tissue damage inflicted by catheter insertion. Manipulation and catheter insertion in the liver shortly before obtaining baseline measures may also explain why circulating ALT was higher at baseline than at the end of the study.

\section{Conclusions}

In conclusion, we found some evidence supporting that low-dose nebulized $\mathrm{NaNO}_{2}$ or inhaled $\mathrm{CO}$ may safely limit intestinal reperfusion injury in the setting of acute hemorrhagic shock. These data also suggest that such protective effects may be secondary to their interaction with mitochondrial respiration, although other mechanisms cannot be excluded. Both nebulized $\mathrm{NaNO}_{2}$ or inhaled $\mathrm{CO}$ hold promise as potential therapeutic adjuncts in the treatment of severe hemorrhagic shock. However, future studies will need to evaluate if these short-term effects translate into improved organ function and recovery. 


\section{Key messages}

- Low doses of nitrite or carbon monoxide may protect intestine from reperfusion injury when inhaled during hemorrhagic shock

- The effects of nitrite and carbon monoxide are probably exerted at a mitochondrial level

- Low doses of inhaled nebulized nitrite or carbon monoxide have no effects on macrohemodynamic parameters

- Microdialysis catheters allow investigating metabolic changes at organ or tissue level

\begin{abstract}
Abbreviations
ADP: adenosine diphosphate; ALT: alanine aminotransferase; AMPK: adenosine monophosphate-activated protein kinase; AST: alanine aminotransferase; ATP: adenosine triphosphate; BW: body weight; CCO: cardiac output; Cl: confidence interval; $\mathrm{CO}$ : carbon monoxide; $\mathrm{COHb}$ : carboxyhemoglobin; e.f.e.: estimated fixed effect; eNOS: endothelial nitrous oxide synthase; $\mathrm{FiO}_{2}$ : fraction of inspired oxygen; HR: heart rate; LPR: lactate to pyruvate ratio; MAP: mean arterial pressure; METHb: methemoglobin; MPAP: mean pulmonary arterial pressure; NADH: nicotinamide adenine dinucleotide dehydrogenase; NADPH: nicotinamide adenine dinucleotide phosphate; $\mathrm{NaNO}_{2}$ : sodium nitrite; NO: nitrous oxide; RCR: respiratory control ratio; RNS: reactive nitrogen species; ROS: reactive oxygen species; $\mathrm{S}_{\mathrm{V}} \mathrm{O}_{2}$ : mixed venous oxygen saturation; SW: stroke volume variation; TBV: total blood volume; wt.: weight.

\section{Competing interests}

Håkon Haugaa, Hernando Gomez, Donald R. Maberry, Andre Holder, Olufunmilayo Ogundele, Ana Maria Botero, Daniel Escobar, Cameron Dezfulian, Elizabeth Kenny, Sruti Shiva, Tor Inge Tønnessen, Brian Zuckerbraun, and Michael R. Pinsky declare no conflicts of interest.
\end{abstract}

\section{Authors' contributions}

$\mathrm{HH}$ contributed to designing the microdialysis part of the experiment, inserting microdialysis catheters, collection and analyses of microdialysis samples, performing statistical analyses, creating figures, and the writing of the manuscript. HG contributed to designing all parts of the study, doing surgery including insertion of microdialysis catheters, collecting hemodynamic data, and the writing of the manuscript. DRM contributed to collecting and analyzing microdialysis samples, doing surgical procedures including insertion of microdialysis catheters, and the writing of the manuscript. $\mathrm{AH}, \mathrm{OO}, \mathrm{AMBQ}$, and $\mathrm{DE}$ contributed to designing the study, collection of hemodynamic data, doing surgical procedures, and the writing of the manuscript. TIT contributed to designing the microdialysis part of the experiment; he financed the microdialysis experiments, and contributed to making the figures and the writing of the manuscript. HA and EK contributed to analyzing nitrite in plasma and microdialysis samples, and the writing of the manuscript. CD contributed to designing, analyzing, and financing the nitrite part of the experiment, and the writing of the manuscript. SS contributed to designing, analyzing, financing the mitochondrial function measurements, and the writing of the manuscript. BZ contributed to designing the study, financed the presence of coworkers (AMBQ and DE) and the pigs, and the writing of the manuscript. MRP contributed to designing all parts of the experiments, provided laboratory facilities, did surgical procedures, and contributed actively in all parts of preparation of the manuscript. All authors read and approved the manuscript.

\section{Funding}

Supported by US Department of Defense grant DM102439 CDMRP and National Institutes of Health grants HL67101, HL07820, HL109068, and 1K12HL109068-02, and South-Eastern Norwegian Health Authorities grants 2012058 and 2013121. Supported by the Center for Metabolism and Mitochondrial Medicine (C3M).

\section{Author details}

'Department of Critical Care Medicine, Cardiopulmonary Research Laboratory, University of Pittsburgh, 3501 Fifth Avenue, Pittsburgh, PA 15260, USA. ${ }^{2}$ Department of Emergencies and Critical Care, Oslo University Hospital, Sognsvannsveien 27 0424, Oslo, Norway. Institute of Clinical Medicine, University of Oslo, Sognsvannsveien 20 0424, Oslo, Norway. ${ }^{4}$ Department of Surgery, University of Pittsburgh, 3380 Boulevard of the Allies 390, Pittsburgh, PA 15213, USA. ${ }^{5}$ Department of Critical Care Medicine, Safar Center for Resuscitation Research University of Pittsburgh, 3550 Terrace Street, Pittsburgh, PA 15261, USA. 'Department of Pharmacology and Chemical Biology, Vascular Medicine Institute, Center for Metabolism and Mitochondrial Medicine, University of Pittsburgh, 200 Lothrop Street, Pittsburgh, PA 15261, USA. ${ }^{7}$ Center for Critical Care Nephrology, University of Pittsburgh, 3550 Terrace Street, Pittsburgh, PA 15261, USA.

Received: 9 December 2014 Accepted: 30 March 2015 Published online: 22 April 2015

\section{References}

1. Hasler RM, Nuesch E, Juni P, Bouamra O, Exadaktylos AK, Lecky F. Systolic blood pressure below $110 \mathrm{~mm} \mathrm{Hg}$ is associated with increased mortality in blunt major trauma patients: multicentre cohort study. Resuscitation. 2011:82:1202-7.

2. Shoemaker WC, Appel PL, Kram HB, Waxman K, Lee TS. Prospective trial of supranormal values of survivors as therapeutic goals in high-risk surgical patients. Chest. 1988:94:1176-86.

3. Boyd O, Grounds RM, Bennett ED. A randomized clinical trial of the effect of deliberate perioperative increase of oxygen delivery on mortality in high-risk surgical patients. JAMA. 1993;270:2699-707.

4. Gan TJ, Soppitt A, Maroof M, El-Moalem H, Robertson KM, Moretti E. Goal-directed intraoperative fluid administration reduces length of hospital stay after major surgery. Anesthesiology. 2002;97:820-6.

5. Eltzschig HK, Eckle T. Ischemia and reperfusion-from mechanism to translation. Nat Med. 2011;17:1391-401.

6. Mesquida J, Masip J, Gili G, Artigas A, Baigorri F. Thenar oxygen saturation measured by near infrared spectroscopy as a noninvasive predictor of low central venous oxygen saturation in septic patients. Intensive Care Med. 2009;35:1106-9.

7. Hernandez G, Bruhn A, Luengo C, Regueira T, Kattan E, Fuentealba A, et al. Effects of dobutamine on systemic, regional and microcirculatory perfusion parameters in septic shock: a randomized, placebo-controlled, double-blind, crossover study. Intensive Care Med. 2013;39:1435-43.

8. Gladwin MT, Raat NJ, Shiva S, Dezfulian C, Hogg N, Kim-Shapiro DB, et al. Nitrite as a vascular endocrine nitric oxide reservoir that contributes to hypoxic signaling, cytoprotection, and vasodilation. Am J Physiol Heart Circ Physiol. 2006;291:H2026-35.

9. Alef MJ, Tzeng E, Zuckerbraun BS. Nitric oxide and nitrite-based therapeutic opportunities in intimal hyperplasia. Nitric Oxide. 2012;26:285-94.

10. Hotchkiss RS, Strasser A, McDunn JE, Swanson PE. Cell death. N Engl J Med 2009:361:1570-83.

11. Clementi E, Brown GC, Feelisch M, Moncada S. Persistent inhibition of cell respiration by nitric oxide: crucial role of S-nitrosylation of mitochondrial complex I and protective action of glutathione. Proc Natl Acad Sci U S A. 1998:95:7631-6.

12. Burwell LS, Nadtochiy SM, Tompkins AJ, Young S, Brookes PS. Direct evidence for S-nitrosation of mitochondrial complex I. Biochem J. 2006:394:627-34

13. Dahm CC, Moore K, Murphy MP. Persistent S-nitrosation of complex I and other mitochondrial membrane proteins by S-nitrosothiols but not nitric oxide or peroxynitrite: implications for the interaction of nitric oxide with mitochondria. J Biol Chem. 2006;281:10056-65.

14. Shiva S, Sack MN, Greer JJ, Duranski M, Ringwood LA, Burwell L, et al. Nitrite augments tolerance to ischemia/reperfusion injury via the modulation of mitochondrial electron transfer. J Exp Med. 2007:204:2089-102.

15. Zuckerbraun BS, Chin BY, Bilban M, d'Avila JC, Rao J, Billiar TR, et al. Carbon monoxide signals via inhibition of cytochrome c oxidase and generation of mitochondrial reactive oxygen species. FASEB J. 2007;21:1099-106.

16. Zuckerbraun BS, Shiva S, Ifedigbo E, Mathier MA, Mollen KP, Rao J, et al. Nitrite potently inhibits hypoxic and inflammatory pulmonary arterial hypertension and smooth muscle proliferation via xanthine oxidoreductase-dependent nitric oxide generation. Circulation. 2010;121:98-109. 
17. Zuckerbraun BS, McCloskey CA, Gallo D, Liu F, Ifedigbo E, Otterbein LE, et al. Carbon monoxide prevents multiple organ injury in a model of hemorrhagic shock and resuscitation. Shock. 2005;23:527-32.

18. Ungerstedt U. Microdialysis-principles and applications for studies in animals and man. J Intern Med. 1991;230:365-73.

19. Haugaa $H$, Thorgersen EB, Pharo A, Boberg KM, Foss A, Line PD, et al. Early bedside detection of ischemia and rejection in liver transplants by microdialysis. Liver Transpl. 2012;18:839-49.

20. Gomez H, Mesquida J, Hermus L, Polanco P, Kim HK, Zenker S, et al. Physiologic responses to severe hemorrhagic shock and the genesis of cardiovascular collapse: can irreversibility be anticipated? I Surg Res. 2012;178:358-69.

21. MacArthur PH, Shiva S, Gladwin MT. Measurement of circulating nitrite and S-nitrosothiols by reductive chemiluminescence. J Chromatogr B Analyt Technol Biomed Life Sci. 2007:851:93-105.

22. Pang L, Wu Y, Dong N, Xu DH, Wang DW, Wang ZH, et al. Elevated serum ubiquitin C-terminal hydrolase-L1 levels in patients with carbon monoxide poisoning. Clin Biochem. 2014;47:72-6.

23. Katz AM, Messineo FC. Lipid-membrane interactions and the pathogenesis of ischemic damage in the myocardium. Circ Res. 1981;48:1-16.

24. Isaksson B, D'souza MA, Jersenius U, Ungerstedt J, Lundell L, Permert J, et al. Continuous assessment of intrahepatic metabolism by microdialysis during and after portal triad clamping. J Surg Res. 2011;169:214-9.

25. Lang Jr JD, Teng X, Chumley P, Crawford JH, Isbell TS, Chacko BK, et al. Inhaled NO accelerates restoration of liver function in adults following orthotopic liver transplantation. J Clin Invest. 2007;117:2583-91.

26. Ingram TE, Fraser AG, Bleasdale RA, Ellins EA, Margulescu AD, Halcox JP, et al. Low-dose sodium nitrite attenuates myocardial ischemia and vascular ischemia-reperfusion injury in human models. J Am Coll Cardiol. 2013;61:2534-41.

27. Li W, Meng Z, Liu Y, Patel RP, Lang JD. The hepatoprotective effect of sodium nitrite on cold ischemia-reperfusion injury. J Transplant. 2012;2012:635179.

28. Dezfulian C, Shiva S, Alekseyenko A, Pendyal A, Beiser DG, Munasinghe JP, et al. Nitrite therapy after cardiac arrest reduces reactive oxygen species generation, improves cardiac and neurological function, and enhances survival via reversible inhibition of mitochondrial complex I. Circulation. 2009:120:897-905

29. Dezfulian C, Alekseyenko A, Dave KR, Raval AP, Do R, Kim F, et al. Nitrite therapy is neuroprotective and safe in cardiac arrest survivors. Nitric Oxide. 2012;26:241-50

30. Kelpke SS, Chen B, Bradley KM, Teng X, Chumley P, Brandon A, et al. Sodium nitrite protects against kidney injury induced by brain death and improves post-transplant function. Kidney Int. 2012;82:304-13.

31. Kamga PC, Mo L, Quesnelle K, Dagda RK, Murillo D, Geary L, et al. Nitrite activates protein kinase $A$ in normoxia to mediate mitochondrial fusion and tolerance to ischaemia/reperfusion. Cardiovasc Res. 2014;101:57-68.

32. Lei C, Yu B, Shahid M, Beloiartsev A, Bloch KD, Zapol WM. Inhaled nitric oxide attenuates the adverse effects of transfusing stored syngeneic erythrocytes in mice with endothelial dysfunction after hemorrhagic shock. Anesthesiology. 2012;117:1190-202.

33. Chouchani ET, Methner C, Nadtochiy SM, Logan A, Pell VR, Ding S, et al. Cardioprotection by S-nitrosation of a cysteine switch on mitochondrial complex I. Nat Med. 2013;19:753-9.

34. Dezfulian C, Raat N, Shiva S, Gladwin MT. Role of the anion nitrite in ischemia-reperfusion cytoprotection and therapeutics. Cardiovasc Res. 2007;75:327-38

35. Lauer T, Preik M, Rassaf T, Strauer BE, Deussen A, Feelisch M, et al. Plasma nitrite rather than nitrate reflects regional endothelial nitric oxide synthase activity but lacks intrinsic vasodilator action. Proc Natl Acad Sci U S A. 2001;98:12814-9.

36. Kim HS, Loughran PA, Rao J, Billiar TR, Zuckerbraun BS. Carbon monoxide activates NF-kappaB via ROS generation and Akt pathways to protect against cell death of hepatocytes. Am J Physiol Gastrointest Liver Physiol. 2008;295:G146-52.

37. Kanagawa F, Takahashi T, Inoue $\mathrm{K}$, Shimizu H, Omori E, Morimatsu $\mathrm{H}$, et al. Protective effect of carbon monoxide inhalation on lung injury after hemorrhagic shock/resuscitation in rats. J Trauma. 2010;69:185-94.

38. Carchman EH, Whelan S, Loughran P, Mollen K, Stratamirovic S, Shiva S, et al. Experimental sepsis-induced mitochondrial biogenesis is dependent on autophagy, TLR4, and TLR9 signaling in liver. FASEB J. 2013;27:4703-11.
39. Zmijewski JW, Banerjee S, Bae H, Friggeri A, Lazarowski ER, Abraham E. Exposure to hydrogen peroxide induces oxidation and activation of AMP-activated protein kinase. J Biol Chem. 2010;285:33154-64.

40. Haugaa H, Almaas R, Thorgersen EB, Foss A, Line PD, Sanengen T, et al. Clinical experience with microdialysis catheters in pediatric liver transplants. Liver Transpl. 2013:19:305-14.

41. Sommer T, Larsen JF. Intraperitoneal and intraluminal microdialysis in the detection of experimental regional intestinal ischaemia. Br J Surg. 2004;91:855-61.

42. MacFie J, O'Boyle C, Mitchell CJ, Buckley PM, Johnstone D, Sudworth P. Gut origin of sepsis: a prospective study investigating associations between bacterial translocation, gastric microflora, and septic morbidity. Gut. 1999:45:223-8.

43. Waldenstrom A, Ronquist G, Aberg AM, Ahlstrom K, Hauck P, Abrahamsson $P$, et al. Ischaemic preconditioning reduces myocardial calcium overload in coronary-occluded pig hearts shown by continuous in vivo assessment using microdialysis. Clin Physiol Funct Imaging. 2012;32:133-8.

44. Gonzalez NR, Hamilton R, Bilgin-Freiert A, Dusick J, Vespa P, Hu X, et al. Cerebral hemodynamic and metabolic effects of remote ischemic preconditioning in patients with subarachnoid hemorrhage. Acta Neurochir Suppl. 2013:115:193-8.

45. Bjornsson B, Winbladh A, Bojmar L, Trulsson LM, Olsson H, Sundqvist T, et al. Remote or conventional ischemic preconditioning-local liver metabolism in rats studied with microdialysis. J Surg Res. 2012;176:55-62.

46. Winbladh A, Bjornsson B, Trulsson L, Offenbartl K, Gullstrand P, Sandstrom P. Ischemic preconditioning prior to intermittent Pringle maneuver in liver resections. J Hepatobiliary Pancreat Sci. 2012;19:159-70.

47. Carles M, Dellamonica J, Roux J, Lena D, Levraut J, Pittet JF, et al. Sevoflurane but not propofol increases interstitial glycolysis metabolites availability during tourniquet-induced ischaemia-reperfusion. $\mathrm{Br} J$ Anaesth. 2008;100:29-35

48. Contaldo C, Harder Y, Plock J, Banic A, Jakob SM, Erni D. The influence of local and systemic preconditioning on oxygenation, metabolism and survival in critically ischaemic skin flaps in pigs. J Plast Reconstr Aesthet Surg. 2007;60:1182-92.

49. Kavianipour M, Ronquist G, Wikstrom G, Waldenstrom A. Ischaemic preconditioning alters the energy metabolism and protects the ischaemic myocardium in a stepwise fashion. Acta Physiol Scand. 2003;178:129-37.

\section{Submit your next manuscript to BioMed Central and take full advantage of:}

- Convenient online submission

- Thorough peer review

- No space constraints or color figure charges

- Immediate publication on acceptance

- Inclusion in PubMed, CAS, Scopus and Google Scholar

- Research which is freely available for redistribution 